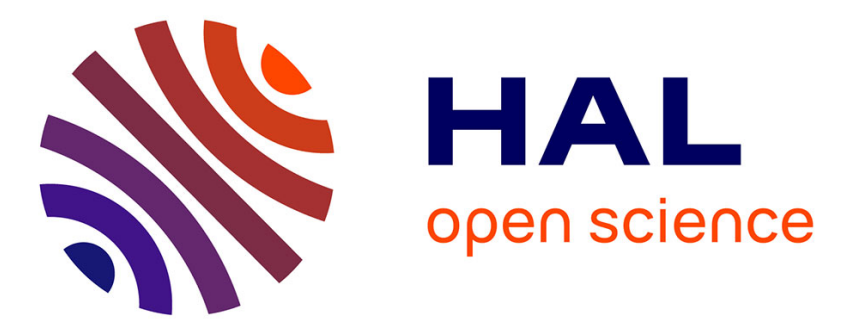

\title{
TRAITEMENT PAR EVAPORATION SOLAIRE DE LIXIVIATS DE DECHETS MENAGERS AU MAROC
}

Fatima Benyoucef, Souad Baati, Abdehadi Makan, Abderrahman El Ghmari

\section{To cite this version:}

Fatima Benyoucef, Souad Baati, Abdehadi Makan, Abderrahman El Ghmari. TRAITEMENT PAR EVAPORATION SOLAIRE DE LIXIVIATS DE DECHETS MENAGERS AU MAROC. Environnement, Ingénierie \& Développement, 2021, 1, pp.20-25. 10.46298/eid.2021.7211 . hal-03149389

\section{HAL Id: hal-03149389 \\ https://hal.science/hal-03149389}

Submitted on 23 Feb 2021

HAL is a multi-disciplinary open access archive for the deposit and dissemination of scientific research documents, whether they are published or not. The documents may come from teaching and research institutions in France or abroad, or from public or private research centers.
L'archive ouverte pluridisciplinaire HAL, est destinée au dépôt et à la diffusion de documents scientifiques de niveau recherche, publiés ou non, émanant des établissements d'enseignement et de recherche français ou étrangers, des laboratoires publics ou privés.

\section{(c)(1)}

Distributed under a Creative Commons Attribution| 4.0 International License 
NOTE TECHNIQUE

TRAITEMENT PAR EVAPORATION SOLAIRE DE LIXIVIATS DE DECHETS MENAGERS AU MAROC

\author{
F. BENYOUCEF ${ }^{1}$, S. BAATI ${ }^{1}$, A. MAKAN ${ }^{2}$, A. EL GHMARI ${ }^{1}$
}

1 Equipe de télédétection et SIG appliquées aux Géosciences et à l'environnement. Faculté des Sciences et Techniques de Beni Mellal Maroc, BP 523000 BM, Mél: fatybenyoucef@gmail.com, Tél : 2120667263170

2 Laboratoire Eau et Environnement, Département de Chimie, Faculté des Sciences, Université Chouaib Doukkali, 24000 El Jadida, Maroc

2 ENQUAS Consulting, Bureau de conseil en qualité, sécurité et environnement, 25000 Khouribga, Maroc

Auteur correspondant : F. BENYOUCEF fatybenyoucef@gmail.com, Tél : 2120667263170

\section{RESUME}

L'objectif de notre travail est le développement de la technique d'évaporation pour le traitement des lixiviats générés par le transport des déchets ménagers dans la ville de Kasba Tadla, Maroc. Tenant compte des caractéristiques du site sélectionné qui bénéficie d'un climat chaud avec des températures supérieures à $50^{\circ} \mathrm{C}$ pendant la période estivale, nous avons privilégié un mode de traitement par évaporation forcée. Plusieurs modèles ont été testés pour évaluer l'influence de la position des bacs d'évaporation par rapport au sol, la couverture des bacs d'évaporation par des plaques de verre et leur angle d'inclinaison et la présence ou l'absence de systèmes d'agitation et de ventilation. Les résultats des essais ont été utilisés pour développer un modèle final d'évaporation forcée dans des dispositifs avec des plaques de verre inclinées et un système d'agitation des lixiviats. Ces conditions ont permis de réduire le temps d'évaporation des lixiviats d'environ $50 \%$ par rapport à l'évaporation naturelle et de $30 \%$ par rapport à l'évaporation avec des plaques de verre horizontales. La vitesse d'évaporation est comprise entre 3,06 mm/jour et 10,25 mm/jour pendant la période estivale. Le modèle développé montre les performances et l'efficacité de l'évaporation pour le traitement des lixiviats.

MOTS-CLÉS : Kasba Tadla, Maroc, Déchets ménagers, Lixiviat, Modèle expérimental, Évaporation forcée, Apport solaire

\section{ABSTRACT}

The aim of our work was the development of evaporation techniques for the treatment of leachate from household waste in the city of Kasba Tadla to avoid rejection permanently in nature. Taking into account the characteristics of the selected site which has a warm climate with temperatures that exceed $50^{\circ} \mathrm{C}$ during the summer period we favored a forced evaporation processing mode. Several models have been tested, the alternatives given on the position of the evaporation pans relative to the ground, coverage evaporation pans by glass plates and their angle of inclination and the presence or absence of systems agitation and ventilation. The tests were used to develop a final evaporation model forced with glass plates inclined with a stirring system of leachate. forced evaporation of leachate testing have helped reduce the time of evaporation of leachate by $50 \%$ approximately relative to natural evaporation and $30 \%$ compared to evaporation with plates of horizontal glass. The power evaporating of this technique from a value of $3.06 \mathrm{~mm} /$ day to reach a value of $10.25 \mathrm{~mm} /$ day during the summer period. The model developed shows evaporation performance and effectiveness for the treatment of leachate so it stands out as alterative techniques for the treatment of leachate.

KEYWORDS: KasbaTadla, Household waste, Leachate, Experimental Model, Forced evaporation, summer period 


\section{INTRODUCTION}

Cette note technique porte sur le traitement des lixiviats générés par la collecte et le transport des déchets ménagers depuis la ville de Kasba Tadlaau Maroc vers le centre d'enfouissement de la ville. Les lixiviats sont des effluents potentiellement toxiques par leur composition complexe et leurs variations temporelles, comme l'ont montré de nombreuses études dans le monde (Le Coupannec et al. 1999, Schlumpf et al. 2001, Kurniawan et al.2006 ; Gourdon et al., 1989). Les études de la littérature internationale ont été essentiellement réalisées sur des lixiviats produits par les déchets dans leurs conditions d'enfouissement en fin de vie. Nos travaux ont porté sur les lixiviats générés pendant le transport des déchets depuis leur lieu de production jusqu'au centre d'enfouissement. La charge organique de ces lixiviats est généralement élevée (Farah Nazet et al. 2012) et les traitements conventionnels d'épuration ne permettent pas toujours de respecter les normes de rejet.

Les techniques de traitement des lixiviats sont très diverses. Les techniques biologiques sont les plus couramment utilisées pour les lixiviats de déchets ménagers, surtout pour les décharges jeunes en cours d'exploitation (Khattabi et al. 2002, Tänia et al. 2013). Elles présentent certaines limites cependant, notamment durant les périodes d'hiver. Compte tenu du fort ensoleillement de la région, la technique par évaporation solaire forcée a été jugée potentiellement intéressante et fait l'objet du présent article. Les conditions climatiques de la zone d'étude se caractérisent par un climat semiaride avec des températures très élevées durant les périodes d'été.

La technique d'évaporation dans des casiers traditionnels au sol est utilisée dans la majorité des décharges contrôlées au Maroc. Les résultats ne donnent cependant pas entière satisfaction à cause des volumes trop importants de lixiviat à traiter (Nameche et Vasel, 1999). Nos travaux visent donc à améliorer cette technique en caractérisant les facteurs d'influence du traitement par évaporation forcée. L'évaporation des lixiviats a été mise en œuvre par plusieurs auteurs mais généralement en couplage avec d'autres techniques telles que l'osmose inverse ou ultrafiltration (Di Palma et al. 2002, Xu et al 2006, Yamasaki et al. 1996). Des travaux précédents publiés par Vincon-Leite et al (2005) et Hancock et al (2011) ont montré que l'évaporation dépend essentiellement de facteurs météorologiques (intensité du rayonnement solaire, température de l'air et de l'eau, humidité relative et spécifique de l'air, pression atmosphérique et vitesse du vent,) et de facteurs physiques du milieu (évaporation à partir de surfaces d'eau libre ou bien à partir d'un sol par exemple (Vincon-Leite et al. 2005). D’autres études ont confirmé aussi que l'évaporation d'une surface d'eau libre dépend non seulement des propriétés physiques et géométriques de cette surface (profondeur, étendue) mais aussi des caractéristiques chimiques de la solution aqueuse (Hancock.2011). Le rapport surface / volume joue un rôle essentiel sur la capacité de cette dernière à emmagasiner de l'énergie. Si le rapport est élevé, le système sera sensible aux variations météorologiques, tandis que si le rapport est faible, l'inertie thermique sera plus grande. D'autre part, le flux d'évaporation est proportionnel à la surface évaporante ainsi qu'à I'humidité relative de l'air en surface. Les mouvements de convection de l'eau et de l'air favorisent les transferts de chaleur entre la solution et I'air à son contact et rend uniforme les températures dans chaque phase. Des mouvements turbulents accroissent considérablement les échanges de chaleur et de matière entre les phases et accélèrent donc l'évaporation (Zhang and Ramanathan, 1995, Nasr et al. 2011, Yan. 1993).

En se basant sur ces informations nous avons étudié l'effet de certains facteurs d'influence afin de déterminer les conditions opératoires optimales. L'approche expérimentale suivie vise dans un premier temps à déterminer les conditions opératoires appropriées pour différents bacs d'évaporations, puis à comparer dans ces conditions le rendement d'évaporation de chaque bac.

\section{MATERIELS ET METHODES}

\subsection{Site d'étude}

Cette étude a été effectuée sur la ville de Kasba Tadla (Figure 1) située entre la ville de Marrakech et la ville de Fès, qui appartient à la province de Béni Mellal de la région de Tadla Azilal au Maroc.

\subsection{Caractérisation des déchets et des lixiviats de la ville de kasba Tadla}

Les lixiviats utilisés dans cette étude ont été collectés à partir des bennes tasseuses de collecte des déchets ménagers de la ville de Kasba Tadla lors du transport à la décharge.

Les déchets ménagers de la ville de Kasba Tadla sont caractérisés par une humidité de $60 \%$ et une forte teneur en matières organiques $(74 \%$ pondéral des matières sèches) et cette dernière varie selon la nature et la composition des déchets entreposés (Hwang et al. 2012). 


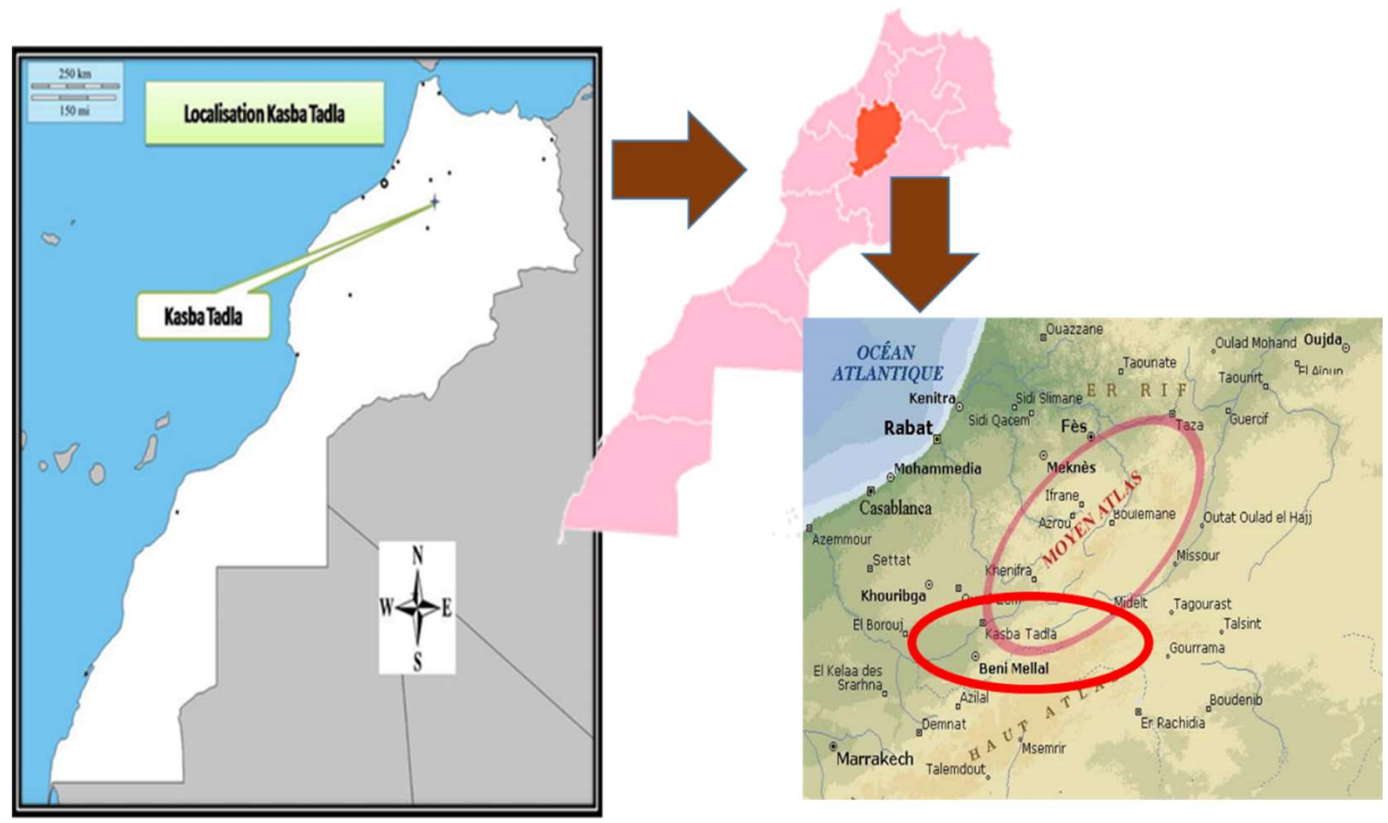

Figure 1 : Cartes de localisation de la ville de Kasba Tadla, Maroc

Les classes de déchets restantes regroupent le papier $(3 \%)$, le plastique ( $9 \%)$, alors que les teneurs en métaux et en verre sont négligeables. Ces résultats sont similaires à ceux trouvés dans d'autres villes du Maroc (Beylot et al. 2013, Ezequiel et al. 2013). Les lixiviats générés par ces déchets ont un $\mathrm{pH}$ d'environ 5 avec une $\mathrm{DCO}$ soluble qui dépasse $20 \mathrm{gO}_{2} / \mathrm{L}$. D'après le suivi effectué sur ces effluents la moyenne journalière de production lors de leurs transports vers la décharge est de l'ordre de 2500 litres par jour par rapport à une quantité collectée des déchets d'environ $40 \mathrm{~T}$ /jours.

\subsection{Dispositif expérimental}

Les essais de traitement ont été réalisés dans des bacs d'évaporation en acier de $93 \mathrm{~cm}$ de longueur, $75 \mathrm{~cm}$ de largeur et $30 \mathrm{~cm}$ de profondeur illustrés à la Fig. 2 . La surface d'évaporation dans chacun des bacs est égale à la section des bacs, soit de l'ordre de $0,7 \mathrm{~m}^{2}$ $(0,93 \times 0,75)$.
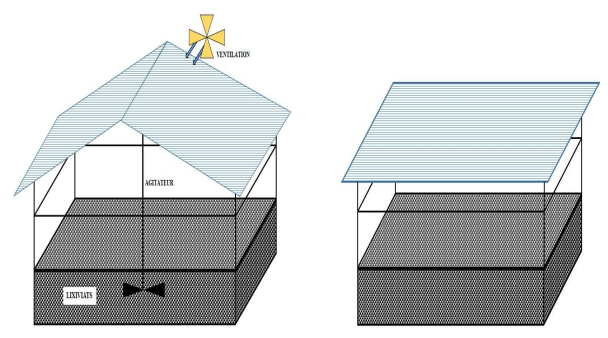

Figure 2. Schémas des dispositifs expérimentaux utilisés (ici bacs avec plaques de verre horizontale ou inclinée)
Chaque bac est rempli au départ de $80 \mathrm{~L}$ de lixiviat, correspondant à une profondeur initiale de l'ordre de $114 \mathrm{~mm}$. L'évaporation est suivie dans le temps par mesures quotidiennes de la hauteur de lixiviat restant dans les bacs. La température est enregistrée pendant toute la durée des essais. Nous avons utilisé en premier lieu deux bacs posés directement à la surface du sol. Le premier est ouvert à l'air libre et l'autre est recouvert d'une plaque de verre posée horizontalement. Puis nous avons utilisé deux autres bacs identiques aux premiers mais posés sur des pieds à une hauteur de $50 \mathrm{~cm}$ au-dessus du sol. Enfin un dernier bac identique aux autres a été utilisé, qui a été posé sur pieds à une hauteur de $50 \mathrm{~cm}$ du sol, recouvert d'un toit fait de deux pans inclinés de vitre, et équipé d'un agitateur.

Les bacs sont posés soit directement sur le sol, soit sur des pieds à $50 \mathrm{~cm}$ du sol. Ils contiennent $80 \mathrm{~L}$ de lixiviat au début des essais (soit une profondeur initiale de l'ordre de $114 \mathrm{~mm}$ ). 


\section{RESULTATS ET DISCUSSIONS}

\subsection{Effet de la surélévation des bacs}

Ces essais ont comparé l'évaporation entre le bac posé au sol et un autre bac de même qualité et de même dimension disposé à côté du premier sur des pieds à 50 $\mathrm{cm}$ du sol. Le seul facteur différenciant les deux essais est donc la surélévation. Les résultats sont illustrés par la Figure 3.

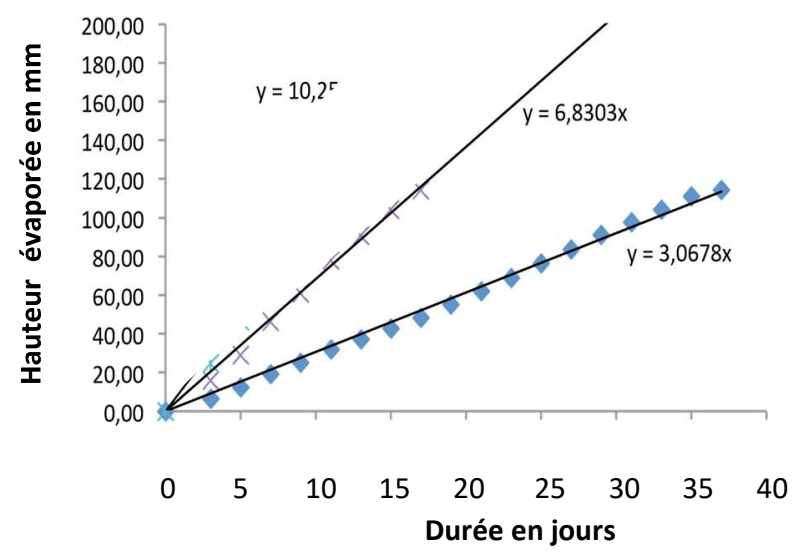

Figure 3 . Evaporation des lixiviats en période estivale dans les bacs non couverts posés directement sur le sol (losanges bleus) ou surélevés de $50 \mathrm{~cm}$ (carrés rouges)

On constate une évolution linéaire de l'évaporation dans les deux configurations comparées, sous une température moyenne de l'ordre de $42^{\circ} \mathrm{C}$.

L'évaporation dans le bac surélevé s'avère plus rapide que celle observée dans le bac posé sur le sol (Fig. 3). Une hauteur de l'ordre de $110 \mathrm{~mm}$ de lixiviat est évaporée en 37 jours lorsque le bac est posé directement sur le sol contre moins de 20 jours dans le bac surélevé. Les deux essais ayant été faits en même temps avec les deux bacs proches l'un de l'autre, les conditions opératoires étaient identiques pour les deux essais. On peut donc penser que la surélévation favorise la circulation de l'air autour du bac et favorise ainsi l'extraction par convection de l'eau évaporée, accélérant ainsi le processus.

\subsection{Effet d'une couverture vitrée horizontale}

Les résultats obtenus, illustrés à la figure 4 indiquent que les plaques vitrées jouent un rôle important dans l'évaporation. Pour les bacs posés au sol, la vitesse moyenne d'évaporation passe de $3,06 \mathrm{~mm} / \mathrm{j}$ sans couverture à $5,27 \mathrm{~mm} / \mathrm{j}$ pour les bacs couverts d'une vitre horizontale, soit une augmentation de $72 \%$. Lorsque les bacs sont surélevés à $50 \mathrm{~cm}$ au-dessus du sol, la vitesse d'évaporation passe de $3,67 \mathrm{~mm} / \mathrm{j}$ (Fig. 3) à $6,83 \mathrm{~mm} / \mathrm{j}$ (Fig. 4), soit une augmentation d'environ $86 \%$. La vitre crée un écran favorable à l'élévation de température par effet de serre à la surface du lixiviat à évaporer. La distance entre la vitre et la surface du bac d'évaporation favorise la ventilation de la surface du lixiviat et améliore ainsi le taux d'évaporation.

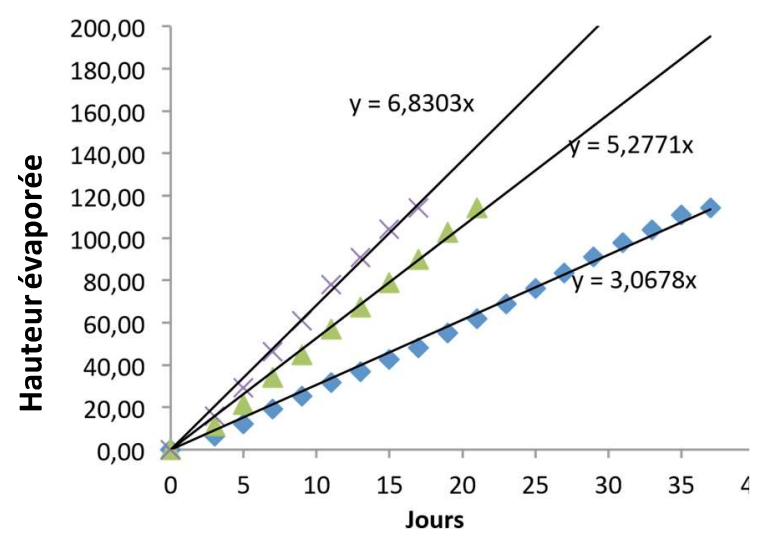

Figure 4 : Effet de la couverture des bacs par une vitre horizontale

Bacs surélevés avec vitre horizontale $(x)$; bacs au sol avec vitre horizontale (triangles) et sans (losanges)

\subsection{Effet d'une couverture vitrée inclinée avec agitation et ventilation}

La Figure 5 montre que l'essai réalisé avec un toit vitré à deux pans inclinés permet d'atteindre une vitesse d'évaporation de 10,25 mm/j en bac surélevé avec agitation et ventilation. Cela correspond à une augmentation de près de $180 \%$ et de $50 \%$ par rapport respectivement à l'essai en bac surélevé non couvert $(3,67 \mathrm{~mm} / \mathrm{j})$ et couvet d'une vitre horizontale $(6,83$ $\mathrm{mm} / \mathrm{j})$. Ces observations montrent que l'inclinaison des plaques vitrées permet d'évacuer l'eau évaporée qui viendrait se condenser sur les vitres alors qu'elle peut retomber dans le bac si la vitre est posée horizontalement. Les vitres inclinées réfléchissent en outre moins de rayonnement incident que les vitres horizontales. La ventilation du ciel gazeux et l'agitation du lixiviat contribuent également à augmenter la vitesse d'évaporation.

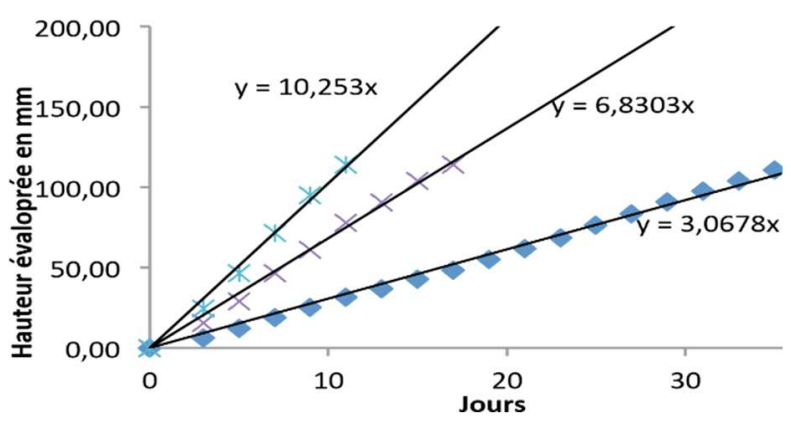

Figure 5 : Effet d'une couverture vitrée horizontale (croix noires) ou inclinée (croix bleues) par rapport à

l'évaporation sans couverture vitrée (losanges) dans les bacs posés directement sur le sol 


\section{CONCLUSION}

Les résultats présentés dans cette note technique montrent l'effet comparé de plusieurs facteurs d'influence de l'évaporation. En premier lieu on observe une augmentation de la vitesse d'évaporation lorsqu' un espace est maintenu entre le sol et la base des bacs. La pose des bacs sur pieds favorise probablement la circulation d'air autour des bacs et donc l'entrainement de l'air humide par convection. La pose d'une plaque vitrée en couverture des bacs avec un espacement suffisant pour permettre la circulation d'air au-dessus de la surface des lixiviats est également un mode opératoire qui accélère l'évaporation, probablement parce qu'elle entraine par effet de serre un échauffement accru de la surface des lixiviats. Une vitre inclinée est préférable à une vitre horizontale pour éviter que l'eau qui se condense sur la vitre ne retombe dans le bac. Enfin, l'agitation des lixiviats évite la formation d'une couche de surface de type lipidique qui vient limiter l'évaporation après quelques jours sans agitation. L'agitation accélère en outre le transfert de chaleur et donc l'évaporation des lixiviats. La combinaison de ces facteurs (surélévation des bacs, couverture vitrée inclinée et agitation) permet de tripler la vitesse d'évaporation par rapport à un bac posé au sol sans couverture vitrée ni agitation.

Le traitement par évaporation est adapté au traitement des lixiviats de la ville de Kasba Tadla, Maroc, du fait des conditions climatiques très favorables, et présente des coûts d'investissement et d'exploitation très bas par rapport à d'autres techniques envisageables.

\section{REFERENCES BIBLIOGRAPHIQUES}

Beylot A et Villeneuve J. (2013). A comparison of 110 French incinerators using a life cycle approach. Waste Management, 33, 2781-2788.

https://doi.org/10.1016/j.wasman.2013.07.00

Di Palma L.Ferrantelli P.,Merli C andPetrucci E. (2002)

Treatment of industrial landfill leachate by means of evaporation and reverse osmosis. Waste Management, 22, 951-955. https://doi.org/10.1016/s0956-053x(02)00079-x

Ezequiel J., Ponce-Ortega J M., Betzabe J., Serna-Gonzalez MandEI-Halwagi M. (2013) Optimal planning for the sustainable utilization of municipal solid waste. Waste Management, 33, 2607-2622.

https://doi.org/10.1016/j.wasman.2013.08.01

Farah Naz A and Christopher Q. (2012) Treatment of landfill leachate using membrane bioreactors: A review.

Desalination, 287, 41-54.

https://doi.org/10.1016/i.desal.2011.12.012

Gourdon, R., Comel, C., Vermande, P. \& Véron, J. (1989) Fractionation of the organic matter of a landfill leachate before and after aerobic or anaerobic biological treatment. Water Research, 23, 167173.
Hancock., M.D. (2011) Indoor swimming pools and leisure centres a model to improve operational effectiveness and reduce environmental impact. CIBSE Technical Symposium, De Montfort University, Leicester, UK, https://doi.org/10.3943/2011.0018

Hwangl.Aoyama H.Matsuto T.,Nakagishi T and Matsuo T.(2012) Recovery of solid fuel from municipal solid waste by hydrothermal treatment using subcritical water. Waste Management, 32, 410-416.

https://doi.org/10.1016/j.wasman.2011.10.00

Khattabi H.,Aleya L et Mania J.(2002) Lagunage naturel de lixiviat de décharge. Sciences de l'eau, 15/1, 411-419. https://doi.org/10.7202/705462ar

Kurniawan A T., Lo W and Chan G. (2006) Physico-chemical treatments for removal of recalcitrant contaminants from landfill leachate. Journal of Hazardous Materials, 129, 80100 https://doi.org/10.1016/i.jhazmat.2005.08.01

Le Coupannec F., Morin D et Peron J J. (1999)

Fractionnement et caractérisation des lixiviats de centre d'enfouissement technique de déchets ménagers. Intérêt de la chromatographie liquide haute performance sur gel d'exclusion stérique Sciences de l'eau, 12/3, 529-543, https://doi.org/10.7202/705364ar

Nameche T et Vasel J L.(1999) Bilan thermique sous climat tempéré des lagunes aérées et naturelles. Sciences de l'eau, 12/1, 65-91, https://doi.org/10.7202/705344ar

Nasr A; Debbissi C; and Ben Nasrallah S.(2011) Evaporation of a binary liquid film by forced convection. Thermal science, 15, 773-784, https://doi.org/10.2298/tsci100427076n

Schlumpf J P.,Trebouet D.,Quemeneur F.,Malleriat J P et Jaouen P.(2001)Réduction de la DCO dure des lisiers de porc et lixiviats par nanofitration. Sciences de l'eau, 14/2, 147-155. https://doi.org/10.7202/705414ar

Tania F.C.V. S., M. Elisabete F. S., A. Cristina C.,Ame'liaF., Isabel S., Sousa M.A., Goncalves C.,AlpenduradaM.F.,BoaventuraR and Vilar V.(2013) Multistage treatment system for raw leachate from sanitary landfill combining biological nitrificationdenitrification/solar photo-Fenton/ biological processes, at a scale close to industrial, Water research, 47, 6167-6186, https://doi.org/10.1016/i.watres.2013.07.036

Vincon-Leite B., Mouchel J M et Tassin B. (2005) Modélisation de l'évolution thermique saisonnière du lac du Bourget (Savoie, France). Sciences de l'eau, 2, 483-510.

https://doi.org/10.7202/705040ar

Xu Y., Yue D., Zhu Y and Nie Y. (2006) Fractionation of dissolved organic matter in mature landfill leachate and its recycling by ultrafiltration and evaporation combined processes.

Chemosphere, 64, 903-911.

https://doi.org/10.1016/i.chemosphere.2006.01.039

Yamasaki A.,Tyaci R. K.,Fouda A and. Matsuura T. (1996) Effect of Evaporation Time on the Pervaporation Characteristics through Homogeneous Aromatic Polyamide Membranes. II. Pervaporation Performances for Ethanol/Water Mixture. Journal of Applied Polymer Science, 60, 743-748, https://doi.org/10.1002/(sici)1097 4628(19960502)60:5\%3C743::aid-app13\%3E3.0.c0;2-\# 
Yan W.M. (1993) Binary diffusion and heat transfer in mixed convection pipe flows with film evaporation. Int. J. Heat Mass Transfer, 36, 2115-2123. https://doi.org/10.1016/s0017$\underline{9310(05) 80142-5}$

Zhang J and Ramanathan V. (1995) Convection-Evaporation Feedback in the Equatorial Pacific, Journal of Climate, 8, 30403051, https://doi.org/10.1175/1520-

0442(1995)008\%3C3040:cefite\%3E2.0.co;2 